\title{
Clinical Factors Associated With the Feeling of Incomplete Bladder Emptying in Women With Little Postvoided Residue
}

\author{
Kwang Suk Lee, Kyo Chul Koo \\ Department of Urology, Yonsei University College of Medicine, Seoul, Korea
}

Purpose: To investigate the clinical factors associated with the feeling of incomplete bladder emptying in female patients with lower urinary tract symptoms (LUTS).

Methods: Records were obtained from a prospectively maintained database for 353 female patients without a history of treatment for LUTS. Patients with comorbidities that may affect voiding function and with postvoid residual (PVR) volume $>20$ $\mathrm{mL}$ or PVR volume-to-bladder capacity ratio of $>10 \%$ were excluded. Finally, 211 patients were eligible for the study.

Results: Patients were stratified according to the severity of the feeling of incomplete emptying: non-severe group ( $\mathrm{n}=147$, $69.7 \%)$ and severe group $(\mathrm{n}=64,30.3 \%)$. The severe group showed longer time to maximum flow rate (Qmax) and greater total overactive bladder symptom score (OABSS). No differences in age, PVR volume, and diagnosis of overactive bladder $(\mathrm{OAB})$ were found between the 2 severity groups. Multivariable analysis identified the severity of the feeling of incomplete emptying to be significantly associated with time to Qmax and minimum volume of voids. Time to Qmax was identified as a significant predictor of severe symptoms in women with OAB. In addition, the feeling of incomplete emptying, total OABSS, and minimum volume of voids were significantly associated with quality of life.

Conclusions: Female patients with the feeling of incomplete emptying who have insignificant PVR volume exhibited severe voiding symptoms and low quality of life. The treatment strategy should be focused on improving the minimum volume of voids and shortening the time to Qmax.

Keywords: Female; Urination disorders; Urinary tract physiological processes; Urinary tract physiological phenomenon

- Research Ethics: The Institutional Review Board of Gangnam Severance Hospital approved this study protocol (approval number: Gangnam Severance Hospital 2019-0467-001). This study was performed with the use of the methodological index for nonrandomized studies. The requirement for informed consent from the patients before study participation was waived.

- Conflict of Interest: No potential conflict of interest relevant to this article was reported.

\section{INTRODUCTION}

Lower urinary tract symptoms (LUTS) are classified into storage, voiding, and postmicturition symptoms (a feeling of incomplete emptying following urination and/or postmicturition leakage) [1]. The feeling of incomplete emptying adopted by the International Continence Society is defined as the subjective sensation or complaint that the bladder does not feel empty at the end of micturition [2]. The feeling of incomplete emptying is seen frequently in both men and women, usually alongside other voiding, storage, or postmicturition LUTS. The feeling of incomplete emptying does not always correlate with the finding of significant residual urine, as it can be felt even with an empty bladder [3].

Symptoms associated with female voiding dysfunction are
Corresponding author: Kyo Chul Koo (iD https://orcid.org/0000-0001-7303-6256 Department of Urology, Yonsei University College of Medicine, 50 Yonsei-ro, Seodaemun-gu, Seoul 03722, Korea

E-mail: GCKOO@yuhs.ac

Submitted: December 29, 2019 / Accepted after revision: February 8, 2020
This is an Open Access article distributed under the terms of the Creative Commons Attribution Non-Commercial License (https://creativecommons.org/licenses/by-nc/4.0/) which permits unrestricted non-commercial use, distribution, and reproduction in any medium, provided the original work is properly cited. 
commonly associated with postmicturition symptoms and women may experience concomitant stress and urge incontinence [4]. Among the postmicturition symptoms in women, the feeling of incomplete voiding is reported to be more common than postvoid dribbling [5]. It is reported that $8.5 \%$ of women have had the experience of the feeling of incomplete emptying. The prevalence is known to increase at the age of 40 years and remains at a steady level of $10 \%$ in older individuals. Overall, several studies have focused on voiding or storage symptoms, whereas the feeling of incomplete voiding has received relatively little attention [6,7].

Uroflowmwtry (UFM) and postvoid residual (PVR) volume are recommended as indispensable first-line objective screening tools [4]. In a previous study analyzing conventional parameters, including voided volume, maximum flow rate (Qmax), and PVR volume, these factors were not associated with the feeling of incomplete emptying in men [8]. Therefore, the present study utilized voiding time, time to Qmax, average flow rate, and flow time in addition to conventional parameters in the analysis of the manifestation of a feeling of incomplete emptying in women. In addition, a 24-hour voiding diary was assessed to analyze the patients' voiding pattern. The primary endpoint of this study was to identify the factors reflecting the feeling of incomplete bladder emptying in women, which may be utilized to develop novel treatment strategies.

\section{MATERIALS AND METHODS}

\section{Patient Cohort}

Medical records were obtained from a prospectively maintained database for female patients without a history of treatment for LUTS between January 2014 and December 2018 at a single outpatient clinic. This cohort was developed to improve the clinical and epidemiological understanding of symptom profiles. The patients' clinicopathologic data, including age, body mass index, LUTS symptoms, UFM, PVR volume, voiding diary, and medical history were obtained. To investigate the chief complaints for visiting our urological outpatient clinic, we provided questionnaires, comprising International Prostate Symptom Score and overactive bladder symptom score (OABSS), to promote the understanding of the components of LUTS. Additionally, the feeling of incomplete emptying, which was assessed according to a score ( 0 , not at all; 1 , less than 1 in times; 2 , less than half the time; 3 , about half the time; 4 , more than half the time; 5 , almost always), was categorized into 2 groups: a score of 0 or 3 as non- severe and a score 4 or 5 as severe [8]. Quality of life was assessed according to a score (0, delighted; 1 , pleased; 2 , mostly satisfied; 3 , mixed; 4 , mostly dissatisfied; 5 , unhappy; 6 , terrible).

The OABSS comprised 4 questions regarding daytime frequency, nocturia, urgency, and urge incontinence. The diagnosis of overactive bladder $(\mathrm{OAB})$ was defined as OABSS $\geq 3$ with an urgency score of $\geq 2$. Using 24 -hour voiding diaries, we reviewed the number of voids per day and night, the minimum and maximum volumes of voids, and the shortest and longest voiding intervals. PVR volume was measured using a bladder scanner (BioSon-500, MCube Tech, Seoul, Korea). Uroflowmetric measurements were performed using Bluetooth UFM (Cube flow_S, MCube Tech, Seoul, Korea). The objective parameters including voided volume (VV), voiding time, average flow rate, Qmax, time to Qmax, and flow time were retrieved from the UFM data.

The detailed medical history of 353 patients was reviewed, and patients with uncontrolled diabetes mellitus, urinary tract infection, urolithiasis, bladder carcinoma, and history of using medications such as hormonal supplements, antipsychotics, or anticholinergics that could affect LUTS, and those who had undergone lower abdominal surgery were excluded $(n=11)$. Patients with a PVR volume $>20 \mathrm{~mL}$ or the ratio of PVR volume to total volume (TV: sum of VV and PVR volume) $>10 \%$ were also excluded $(\mathrm{n}=131)$.

\section{Good Clinical Practice Protocols}

The study was carried out in agreement with applicable laws and regulations, good clinical practices, and ethical principles as described in the Declaration of Helsinki. The Institutional Review Board of Gangnam Severance Hospital approved this study protocol (approval number: Gangnam Severance Hospital 2019-0467-001). This study was performed with the use of the methodological index for nonrandomized studies. The requirement for informed consent from the patients before study participation was waived.

\section{Statistical Analyses}

All of the values were expressed as number (\%) or mean \pm standard deviation. Between patient groups, the parameters were compared using Student t-test. Simple and multiple linear regression analyses were used to identify the independent parameters associated with the feeling of incomplete emptying or quality of life. Univariable and multivariable analyses were performed to predict the group with severe symptoms. Receiver operating characteristic (ROC) 
curves and area under the ROC curves were used to obtain the cutoff value. These optimal cutoff values were based on predefined values and according to a sensitivity analysis using Youden index (sensitivity+specificity -1 ). All reported P-values are 2-sided, and a P-value of $\leq 0.05$ was considered to indicate statistical significance. All statistical tests were carried out with IBM SPSS Statistics ver. 25.0 (IBM Co., Armonk, NY, USA).

\section{RESULTS}

Differences in Clinical Features and Voiding Patterns According to the Severity of the Feeling of Incomplete Emptying

Among the 211 patients (mean score for the feeling of incomplete emptying $=2.6 \pm 1.6), 147$ patients $(69.7 \%)$ experienced non-severe symptoms (non-severe group), and 64 (30.3\%) had

Table 1. Differences in clinical features according to symptom severity based on the feeling of incomplete emptying

\begin{tabular}{|c|c|c|c|c|}
\hline Variable & Total & Non-severe $(0-3)$ & Severe (4-5) & P-value \\
\hline No. of patients & 211 & $147(69.7)$ & $64(30.3)$ & \\
\hline Age (yr) & $57.0 \pm 13.9$ & $56.3 \pm 14.6$ & $58.7 \pm 12.1$ & 0.220 \\
\hline $\begin{array}{l}\text { Voiding symptoms } \\
\text { Residual urine sense } \\
\text { Frequency } \\
\text { Urgency } \\
\text { Intermittency } \\
\text { Weak urine stream } \\
\text { Hesitancy } \\
\text { Nocturia }\end{array}$ & $\begin{array}{r}83(39.3) \\
114(54.0) \\
80(37.9) \\
58(27.5) \\
83(39.3) \\
26(12.3) \\
54(25.6)\end{array}$ & $\begin{array}{l}30(20.4) \\
77(52.4) \\
60(40.8) \\
29(19.7) \\
51(34.7) \\
16(10.6) \\
42(28.6)\end{array}$ & $\begin{array}{l}53(82.8) \\
37(57.8) \\
20(31.3) \\
29(45.3) \\
32(50.0) \\
10(15.6) \\
12(18.8)\end{array}$ & $\begin{array}{r}<0.001 \\
0.469 \\
0.181 \\
<0.001 \\
0.042 \\
0.338 \\
0.134\end{array}$ \\
\hline $\mathrm{TV}(\mathrm{mL})$ & $201.8 \pm 143.3$ & $205.7 \pm 144.0$ & $192.9 \pm 142.4$ & 0.551 \\
\hline PVR volume-to-TV ratio (\%) & $2.8 \pm 2.8$ & $2.9 \pm 2.8$ & $2.8 \pm 2.9$ & 0.864 \\
\hline PVR volume (mL) & $5.3 \pm 5.5$ & $5.2 \pm 5.1$ & $5.6 \pm 6.4$ & 0.651 \\
\hline Voided volume (mL) & $196.5 \pm 141.1$ & $200.5 \pm 142.3$ & $187.3 \pm 138.9$ & 0.532 \\
\hline Voiding time (sec) & $24.5 \pm 16.3$ & $23.6 \pm 16.0$ & $26.5 \pm 17.0$ & 0.238 \\
\hline $\mathrm{Qmax}(\mathrm{mL} / \mathrm{sec})$ & $19.6 \pm 10.4$ & $19.7 \pm 10.3$ & $19.2 \pm 10.6$ & 0.740 \\
\hline Time to Qmax (sec) & $8.1 \pm 5.8$ & $7.4 \pm 4.6$ & $9.7 \pm 7.6$ & 0.028 \\
\hline Average flow rate (mL/sec) & $10.3 \pm 6.5$ & $10.8 \pm 7.0$ & $9.0 \pm 4.7$ & 0.063 \\
\hline Flow time (sec) & $20.6 \pm 11.9$ & $20.3 \pm 12.0$ & $21.4 \pm 11.9$ & 0.552 \\
\hline No. of voids per $24 \mathrm{hr}$ & $11.4 \pm 4.0$ & $11.2 \pm 3.9$ & $11.8 \pm 4.3$ & 0.324 \\
\hline Minimum volume of voids (mL) & $64.7 \pm 47.1$ & $66.5 \pm 48.0$ & $60.6 \pm 45.0$ & 0.402 \\
\hline Maximum volume of voids (mL) & $362.5 \pm 209.3$ & $373.3 \pm 226.7$ & $337.5 \pm 161.2$ & 0.254 \\
\hline Shortest voiding interval (min) & $61.6 \pm 36.0$ & $61.2 \pm 35.4$ & $62.6 \pm 37.6$ & 0.793 \\
\hline Longest voiding interval (min) & $320.7 \pm 117.1$ & $326.8 \pm 119.0$ & $306.6 \pm 112.2$ & 0.252 \\
\hline No. of voids per night & $2.1 \pm 1.4$ & $2.0 \pm 1.4$ & $2.2 \pm 1.3$ & 0.417 \\
\hline $\mathrm{OAB}$ & $152(72.0)$ & $108(73.5)$ & $44(68.8)$ & 0.485 \\
\hline Total OABSS & $7.1 \pm 3.6$ & $6.5 \pm 3.1$ & $8.3 \pm 4.3$ & 0.004 \\
\hline Daytime frequency & $0.9 \pm 0.6$ & $0.8 \pm 0.6$ & $1.2 \pm 0.6$ & 0.001 \\
\hline Nocturia & $2.1 \pm 1.0$ & $1.9 \pm 1.0$ & $2.4 \pm 0.8$ & 0.001 \\
\hline Urgency & $2.7 \pm 1.7$ & $2.5 \pm 1.5$ & $2.9 \pm 1.9$ & 0.165 \\
\hline Urge incontinence & $1.4 \pm 1.8$ & $1.2 \pm 1.6$ & $1.9 \pm 2.0$ & 0.031 \\
\hline Urinary incontinence & $55(26.1)$ & $33(22.4)$ & $22(34.4)$ & 0.034 \\
\hline
\end{tabular}

Values are presented as number (\%) or mean \pm standard deviation.

TV, total volume; PVR, postvoid residual; Qmax, maximum flow rate; OAB, overactive bladder; OABSS, overactive bladder symptom score. 
severe symptoms (severe group). OAB was diagnosed in 152 patients (72.0\%). The severe group exhibited significantly longer time to Qmax, greater total OABSS, daytime frequency, nocturia, and urge incontinence. However, there were no differences in age, PVR volume, PVR volume-to-TV ratio, VV, voiding time, Qmax, and the diagnosis of OAB between the severe and non-severe groups (Table 1).

The feeling of incomplete emptying was significantly associated with time to $\mathrm{Qmax}(\beta=0.04 ; 95 \%$ confidence interval $[\mathrm{CI}]$, $0.007-0.081 ; \mathrm{P}=0.019)$ and minimum volume of voids $(\beta=-0.01$; $95 \% \mathrm{CI},-0.010$ to $-0.001 ; \mathrm{P}=0.026$ ). In contrast, the feeling of incomplete emptying was not related to total OABSS, daytime frequency, nocturia, and urge incontinence (Table 2). In the univariable analysis, time to Qmax was identified as the only significant factor predicting the group with severe symptoms (odds ratio [OR], 1.07; 95\% CI, 1.015-1.123; $\mathrm{P}=0.011$ ).

\section{Predictive Factors for the Feeling of Incomplete Emptying in Patients With OAB}

The baseline clinical and demographic characteristics of the pa- tients according to the diagnosis of $\mathrm{OAB}$ are shown in Table 3. The OAB patients showed a higher PVR volume-to-TV ratio, higher number of voids per 24 hours, and shorter longest voiding interval than the non-OAB patients. In terms of age, VV, Qmax, time to Qmax, minimum and maximum volumes of voids, shortest voiding interval, and number of voids per nights, no differences were found between the patients with and without OAB. Time to Qmax ( $\beta=0.04 ; 95 \% \mathrm{CI}, 0.001-0.086 ; \mathrm{P}=0.045)$ and minimum volume of voids ( $\beta=-0.00 ; 95 \% \mathrm{CI},-0.890$ to $0.000 ; \mathrm{P}=0.005$ ) were significantly correlated with the feeling of incomplete emptying in women with OAB (Table 4). The optimal cutoff values of time to $\mathrm{Qmax}$ and minimum volume of voids were $8.9 \mathrm{~s}$ and $45 \mathrm{~mL}$, respectively. Time to Qmax was the only factor predicting a severe feeling of incomplete emptying (OR, 1.07; 95\% CI, 1.011-1.140; $\mathrm{P}=0.020)$ in the univariable analysis. However, for non-OAB women, no parameters were correlated with the severe symptoms.

\section{Assessment of Quality of Life Associated With the Feeling of Incomplete Emptying}

A subanalysis was performed to identify the factors associated

Table 2. Univariable and multivariable linear regression analyses for increasing severity of the feeling of incomplete emptying

\begin{tabular}{|c|c|c|c|c|}
\hline \multirow{2}{*}{ Variable } & \multicolumn{2}{|c|}{ Univariable } & \multicolumn{2}{|c|}{ Multivariable } \\
\hline & $\beta(95 \% \mathrm{CI})$ & P-value & $\beta(95 \% \mathrm{CI})$ & P-value \\
\hline Age & $-0.01(-0.018$ to 0.014$)$ & 0.775 & & \\
\hline TV & $0.00(-0.002$ to 0.001$)$ & 0.553 & & \\
\hline PVR volume-to-TV ratio & $-0.03(-0.105$ to 0.051$)$ & 0.498 & & \\
\hline PVR volume & $0.00(-0.040$ to 0.040$)$ & 0.997 & & \\
\hline Voided volume & $0.00(-0.002$ to 0.001$)$ & 0.547 & & \\
\hline Voiding time & $0.01(-0.002$ to 0.001$)$ & 0.227 & & \\
\hline Qmax & $-0.01(-0.029$ to 0.013$)$ & 0.438 & & \\
\hline Time to Qmax & 0.05 (0.010 to 0.084$)$ & 0.014 & 0.04 (0.007 to 0.081$)$ & 0.019 \\
\hline Average flow rate & $-0.03(-0.064$ to 0.003$)$ & 0.072 & & \\
\hline Flow time & $0.01(-0.013$ to 0.024$)$ & 0.567 & & \\
\hline No. of voids per $24 \mathrm{hr}$ & $0.04(-0.019$ to 0.089$)$ & 0.206 & & \\
\hline Minimum volume of voids & $-0.01(-0.010$ to -0.001$)$ & 0.020 & $-0.01(-0.010$ to -0.001$)$ & 0.026 \\
\hline Maximum volume of voids & $-0.00(-0.002$ to 0.000$)$ & 0.058 & & \\
\hline Shortest voiding interval & $-0.00(-0.007$ to 0.006$)$ & 0.866 & & \\
\hline Longest voiding interval & $-0.00(-0.003$ to 0.000$)$ & 0.111 & & \\
\hline No. of voids per night & 0.07 (-0.086 to 0.228$)$ & 0.374 & & \\
\hline $\mathrm{OAB}$ & $-0.03(-0.513$ to 0.458$)$ & 0.911 & & \\
\hline Urinary incontinence & $0.16(-0.063$ to 0.388$)$ & 0.158 & & \\
\hline
\end{tabular}

$\mathrm{CI}$, confidence interval; TV, total volume; PVR, postvoid residual; Qmax, maximum flow rate; $\mathrm{OAB}$, overactive bladder. 
Table 3. Comparison of the patients with and without the diagnosis of overactive bladder

\begin{tabular}{|c|c|c|c|}
\hline Variable & Non-OAB $(\mathrm{n}=59)$ & $\mathrm{OAB}(\mathrm{n}=152)$ & P-value \\
\hline Age (yr) & $58.5 \pm 14.5$ & $56.4 \pm 13.6$ & 0.321 \\
\hline $\mathrm{TV}(\mathrm{mL})$ & $231.9 \pm 162.7$ & $190.1 \pm 133.7$ & 0.083 \\
\hline PVR volume-to-TV ratio (\%) & $1.8 \pm 2.3$ & $3.3 \pm 2.9$ & $<0.001$ \\
\hline PVR volume (mL) & $3.9 \pm 5.1$ & $5.8 \pm 5.6$ & 0.018 \\
\hline Voided volume (mL) & $228.0 \pm 11.6$ & $184.3 \pm 130.8$ & 0.067 \\
\hline Voiding time (sec) & $25.4 \pm 13.9$ & $24.1 \pm 17.2$ & 0.609 \\
\hline $\operatorname{Qmax}(\mathrm{mL} / \mathrm{sec})$ & $21.3 \pm 12.1$ & $18.9 \pm 9.7$ & 0.139 \\
\hline Time to Qmax (sec) & $8.3 \pm 5.7$ & $8.1 \pm 5.8$ & 0.851 \\
\hline Average flow rate $(\mathrm{mL} / \mathrm{sec})$ & $11.2 \pm 7.2$ & $9.9 \pm 6.1$ & 0.223 \\
\hline Flow time (sec) & $21.1 \pm 9.0$ & $20.5 \pm 12.9$ & 0.712 \\
\hline No. of voids per $24 \mathrm{hr}$ & $10.5 \pm 3.7$ & $11.7 \pm 4.1$ & 0.046 \\
\hline Minimum volume of voids (mL) & $74.4 \pm 50.7$ & $60.9 \pm 45.2$ & 0.061 \\
\hline Maximum volume of voids (mL) & $407.0 \pm 319.4$ & $345.2 \pm 143.7$ & 0.157 \\
\hline Shortest voiding interval (min) & $68.4 \pm 38.8$ & $59.0 \pm 34.6$ & 0.087 \\
\hline Longest voiding interval (min) & $354.8 \pm 138.0$ & $307.4 \pm 105.4$ & 0.019 \\
\hline No. of voids per night & $2.2 \pm 1.6$ & $2.0 \pm 1.3$ & 0.625 \\
\hline Urinary incontinence & $4(6.8)$ & $51(33.6)$ & $<0.001$ \\
\hline
\end{tabular}

Values are presented as mean \pm standard deviation or number (\%).

$\mathrm{OAB}$, overactive bladder; TV, total volume; PVR, postvoid residual; Qmax, maximum flow rate.

Table 4. Univariable and multivariable linear regression analyses for increasing the severity of the feeling of incomplete emptying in patients with overactive bladder

\begin{tabular}{|c|c|c|c|c|}
\hline \multirow{2}{*}{ Variable } & \multicolumn{2}{|c|}{ Univariable } & \multicolumn{2}{|c|}{ Multivariable } \\
\hline & $\beta(95 \% \mathrm{CI})$ & P-value & $\beta(95 \% \mathrm{CI})$ & P-value \\
\hline Age & $-0.01(-0.026$ to 0.012$)$ & 0.460 & & \\
\hline TV & $-0.00(-0.003$ to 0.001$)$ & 0.535 & & \\
\hline PVR volume-to-TV ratio & $-0.03(-0.117$ to 0.062$)$ & 0.548 & & \\
\hline PVR volume & $-0.01(-0.053$ to 0.040$)$ & 0.784 & & \\
\hline Voided volume & $-0.00(-0.003$ to 0.001$)$ & 0.533 & & \\
\hline Voiding time & $0.01(-0.006$ to 0.024$)$ & 0.233 & & \\
\hline Qmax & $-0.01(-0.038$ to 0.015$)$ & 0.388 & & \\
\hline Time to Qmax & 0.05 (0.009 to 0.095$)$ & 0.019 & 0.04 (0.001 to 0.086$)$ & 0.045 \\
\hline Average flow rate & $-0.03(-0.072$ to 0.011$)$ & 0.152 & & \\
\hline Flow time & $0.00(-0.019$ to 0.021$)$ & 0.911 & & \\
\hline No. of voids per $24 \mathrm{hr}$ & $0.06(-0.001$ to 0.122$)$ & 0.054 & & \\
\hline Minimum volume of voids & $-0.01(-0.014$ to -0.003$)$ & 0.003 & $-0.01(-0.013$ to -0.002$)$ & 0.005 \\
\hline Maximum volume of voids & $-0.00(-0.004$ to -0.001$)$ & 0.008 & $-0.00(-0.890$ to 0.000$)$ & 0.118 \\
\hline Shortest voiding interval & $-0.00(-0.011$ to 0.004$)$ & 0.351 & & \\
\hline Longest voiding interval & $-0.00(-0.004$ to 0.001$)$ & 0.122 & & \\
\hline No. of voids per night & $0.06(-0.141$ to 0.256$)$ & 0.567 & & \\
\hline Urinary incontinence & $0.23(-0.020$ to 0.472$)$ & 0.072 & & \\
\hline
\end{tabular}

CI, confidence interval; TV, total volume; PVR, postvoid residual; Qmax, maximum flow rate. 
with the quality of life. The feeling of incomplete emptying, total OABSS, voided volume, and minimum volume of voids were significantly correlated with quality of life $(\beta=0.12$ [95\% CI, 0.048-0.200], $\mathrm{P}=0.001 ; \beta=0.09$ [95\% CI, 0.056-0.124], $\mathrm{P}<0.001$; $\beta=-0.00$ [ $95 \% \mathrm{CI},-0.002$ to 0.000$], P=0.003$; and $\beta=-0.00$ [95\% $\mathrm{CI},-0.006$ to -0.001$], \mathrm{P}=0.011$, respectively). $\mathrm{PVR}$ volume was not correlated with the quality of life $(r=0.027, \mathrm{P}=0.705)$.

\section{DISCUSSION}

Urologists and general practitioners encounter women with LUTS who complain of the feeling of incomplete emptying despite no or little PVR volume in the clinical setting. PVR volume in several urodynamic studies was not useful for the assessment of the feeling of incomplete emptying [9-11]. Furthermore, VV and Qmax in UFM as first-line screening parameters were not correlated with the feeling of incomplete emptying in men [8]. Therefore, to date, the feeling of incomplete emptying has only been regarded as a subjective symptom. For the precise explanation of this symptom, we additionally introduced timedependent parameters as obtained by UFM. Of note, time to Qmax was identified as a predictive factor for patients with severe symptoms. Moreover, we reviewed 24-hour voiding diary data to analyze voiding patterns. Smaller minimum volume of voids was associated with an increase in the severity of feeling of incomplete emptying in patients with $\mathrm{OAB}$.

There are several presumptions that explain the mechanisms underlying the feeling of incomplete emptying in women. First, time-dependent parameters obtained by UFM indicated time to Qmax as a significant factor in patients with severe symptoms. The delay in time to Qmax may have affected the patients' feeling of incomplete emptying regardless of Qmax itself. Second, a smaller minimum volume of voids was associated with increased severity of feeling of incomplete emptying in patients with OAB. Decreased Qmax owing to a small-voided volume, in turn, may have provoked the feeling of incomplete emptying. Such factors assessed by uroflowmetric measurements may indicate multifactorial etiologies underlying the feeling of incomplete bladder emptying in women with little PVR. In addition, experience of small-voided volume may have provoked the feeling of PVR. Therefore, these 2 factors, independently or in combination, may have affected the deterioration of the feeling of incomplete emptying and quality of life.

Urologists and general practitioners have questioned why some patients have the feeling of incomplete emptying. Several explanations for the development of such symptoms have been suggested. First, the feeling of incomplete emptying with little PVR volume may be associated with the sensory changes in the bladder. Afferent activity is derived from some degree of volume, tension, and nociceptive receptors in the bladder and urethra [12]. The bladders of OAB patients appear to be more sensitive than those of non-OAB patients [13-15]. In an analysis of voiding behaviors in $\mathrm{OAB}$ patients, the degree of urgency sensation was increased in $\mathrm{OAB}$ patients compared to that in normal subjects at any given bladder volume, and this was not dependent on the urgency episodes [14]. Van Brummen et al. [13] showed that patients with $\mathrm{OAB}$ symptoms had lower micturition and bladder volumes than those without the symptoms. Therefore, a hypersensitive bladder can explain why some patients have the feeling of incomplete emptying concomitant with $\mathrm{OAB}$ disorder. Second, regarding female sex, pregnancy and parturition might have a delayed effect on the development of the feeling of incomplete emptying. The voiding problems associated with urinary urgency and voiding difficulties in women may be more likely to worsen among those who have had multiple vaginal deliveries due to repeated injury to the muscles, nerves, and connective tissue of the pelvic floor [16]. In rats with multiple pregnancies and parturition, the bladder showed hypertrophy, instabilities, increased voiding pressure, and residual volume [17]. The modified bladder function leads to a dysfunction similar to that induced by obstruction, which increases the sensitivity to adrenergic and cholinergic stimulations compared to that in controls. This assumption supports the findings of Maserejian et al. [5], who reported that the prevalence of postmicturition symptoms increased at the age of 40 years in women.

Our results showed that the etiology of the feeling of incomplete emptying may arise from both voiding and storage mechanisms. The feeling of incomplete emptying was significantly associated with worsening of both voiding and storage symptoms in men, which was consistent with previously reported results [8]. The present work is the first study to identify that total OABSS and daytime frequency were higher and nocturia and urge incontinence were more frequent among women with severe symptoms, whereas urgency in the OABSS subdomain scores had no association with severe symptoms. However, the feeling of incomplete emptying seemed to not be associated with $\mathrm{OAB}$. The proportion of OAB diagnosis was not different between the non-severe and severe groups. Generally, women with bladder oversensitivity exhibit a bladder capacity of $<250$ 
$\mathrm{mL}$ [18]. In this study, there were no differences in the bladder capacities according to the severity of the feeling of incomplete emptying. Therefore, our data suggest that the feeling of incomplete emptying is not related to $\mathrm{OAB}$ or bladder oversensitivity, but instead can be regarded as a separate category.

Women who report the feeling of incomplete emptying among the LUTS subtypes have been reported to be most bothered by their health issues $[5,19,20]$. In our study, the feeling of incomplete emptying was significantly correlated with decreased quality of life, which is comparable to previously published findings. An experimental study in rats with multiple pregnancies and parturition reported that bladder dysfunction showed an increasing sensitivity to adrenergic and cholinergic stimulations [17]. The feeling of incomplete bladder emptying occurs immediately after micturition; thus, it may be postulated that specific neurotransmitters and their receptors would be associated with the symptoms. Physicians are frequently faced with the dilemma of prescribing alpha-blockers or anticholinergics in patients with the feeling of incomplete emptying as its pathophysiology remains unclear. Despite this unclear rationale, our findings would thus be useful in developing novel treatment strategies.

Despite the several strengths, the present study was inherently limited by its small size and retrospective nature. Moreover, although the clinical factors associated with the feeling of incomplete bladder emptying in women were identified, urodynamic studies to measure bladder function are required for precise diagnosis. Furthermore, the criterion used to stratify the severity of feeling of incomplete emptying was arbitrary; thus, it warrants validation in future studies. Finally, although the menopausal status and vaginal birth delivery were associated with the significant factors of female voiding symptoms, we did not check the hormonal therapy history of the patients in our database. Future studies are warranted to elucidate the basic mechanism of incomplete voiding sensation and to design trials to improve the symptoms and quality of life in women with the feeling of incomplete emptying.

In conclusion, women with the feeling of incomplete emptying who have insignificant PVR volume following urination exhibit severe voiding dysfunctions and low quality of life. This symptom may be associated with the small minimum volume of voids and longer time to Qmax, and this finding may be utilized to develop novel treatment strategies.

\section{AUTHOR CONTRIBUTION STATEMENT}

- Conceptualization: $K C K$

- Investigation: $K S L$

- Formal analysis: KSL

- Methodology: KSL

- Project administration: $K C K$

-Writing - original draft: KSL

-Writing - review \& editing: KCK

\section{REFERENCES}

1. Park J, Kim ES, Lee YJ, Lee HS, Seo JT. Sex differences in lower urinary tract symptoms in older Korean adults living in rural areas: prevalence, quality of life, and associated factors. Int Neurourol J 2018;22:212-9.

2. Haylen BT, de Ridder D, Freeman RM, Swift SE, Berghmans B, Lee J, et al. An International Urogynecological Association (IUGA)/International Continence Society (ICS) joint report on the terminology for female pelvic floor dysfunction. Neurourol Urodyn 2010; 29:4-20.

3. Sountoulides P, Mutomba WF. Feeling of incomplete bladder emptying: a definition with clinical implications. Low Urin Tract Symptoms 2019;11:85.

4. Robinson D, Staskin D, Laterza RM, Koelbl H. Defining female voiding dysfunction: ICI-RS 2011. Neurourol Urodyn 2012;31:3136.

5. Maserejian NN, Kupelian V, McVary KT, Doshi M, Link CL, McKinlay JB. Prevalence of post-micturition symptoms in association with lower urinary tract symptoms and health-related quality of life in men and women. BJU Int 2011;108:1452-8.

6. Park YW, Lee JH. Female urinary incontinence and obesity assessed by anthropometry and dual-energy X-ray absorptiometry: Analysis from the 2008-09 Korean National Health and Nutrition Examination Survey. Low Urin Tract Symptoms 2019;11:O28-33.

7. Lee JH, Lee HS. Nutrient intake and urinary incontinence in Korean women: a propensity score-matched analysis from the Korea National Health and Nutrition Examination Survey data. Int J Urol 2017;24:793-7.

8. Lee JY, Lee DH, Lee H, Bang WJ, Hah YS, Cho KS. Clinical implications of a feeling of incomplete emptying with little post-void residue in men with lower urinary tract symptoms. Neurourol Urodyn 2014;33:1123-7.

9. Dietz HP, Haylen BT. Symptoms of voiding dysfunction: what do they really mean? Int Urogynecol J Pelvic Floor Dysfunct 2005;16: 
52-5; discussion 55 .

10. Groutz A, Gordon D, Lessing JB, Wolman I, Jaffa A, David MP. Prevalence and characteristics of voiding difficulties in women: are subjective symptoms substantiated by objective urodynamic data? Urology 1999;54:268-72.

11. Jeffery ST, Doumouchtsis SK, Vlachos IS, Fynes MM. Are voiding symptoms really associated with abnormal urodynamic voiding parameters in women? Int J Urol 2008;15:1044-8.

12. Wein AJ. Pathophysiology and classification of lower urinary tract dysfunction: overview. In: McDougal WS, Wein AJ, Kavoussi LR, Novick AC, Partin AW, Peters CA, et al. Campbell-Walsh urology. 10th ed. Philadelphia (PA): Saunders; 2012. p. 1834-46.

13. Van Brummen HJ, Heintz AP, van der Vaart CH. The association between overactive bladder symptoms and objective parameters from bladder diary and filling cystometry. Neurourol Urodyn 2004; 23:38-42.

14. Yamaguchi O, Honda K, Nomiya M, Shishido K, Kakizaki H, Tanaka H, et al. Defining overactive bladder as hypersensitivity. Neurourol Urodyn 2007;26(6 Suppl):904-7.

15. Park KK, Lee SH, Chung BH, Kim SJ, Kwak C, et al. Efficacy and safety of low-dose anticholinergics to treat men with lower urinary tract symptoms with overactive bladder: a retrospective study based on real life practice. Prostate Int 2013;1:37-41.

16. Handa VL, Harris TA, Ostergard DR. Protecting the pelvic floor: obstetric management to prevent incontinence and pelvic organ prolapse. Obstet Gynecol 1996;88:470-8.

17. Grandadam F, Lluel P, Palea S, Martin DJ. Pharmacological and urodynamic changes in rat urinary bladder function after multiple pregnancies. BJU Int 1999;84:861-6.

18. Abrams P. Urodynamics. 3rd ed. London: Springer-Verlag; 2006. Chapter 5, Urodynamics in clinical practice; 147-70.

19. Kwon CS, Lee JH. Prevalence, risk factors, quality of life, and health-care seeking behaviors of female urinary incontinence: results from the 4th Korean National Health and Nutrition Examination Survey VI (2007-2009). Int Neurourol J 2014;18:31-6.

20. Lee HS, Lee JH. Vitamin D and urinary incontinence among Korean Women: a propensity score-matched analysis from the 20082009 Korean National Health and Nutrition Examination Survey. J Korean Med Sci 2017;32:661-5. 